
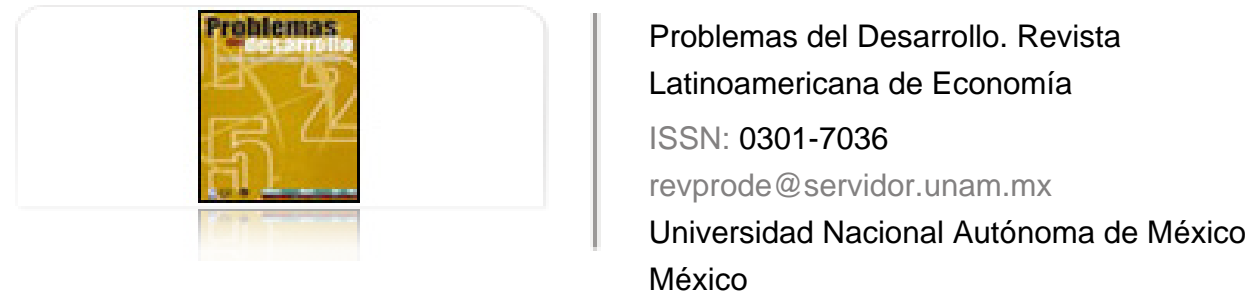

Mendoza Cota, Jorge Eduardo; García Bermúdez, Karina Jazmín

Discriminación salarial por género en México

Problemas del Desarrollo. Revista Latinoamericana de Economía, vol. 40, núm. 156, enero-marzo, 2009, pp. 77-99

Universidad Nacional Autónoma de México

Distrito Federal, México

Disponible en: http://www.redalyc.org/articulo.oa?id=11820096006

- Cómo citar el artículo

- Número completo

- Más información del artículo

Página de la revista en redalyc.org

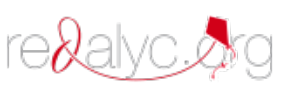

Sistema de Información Científica

Red de Revistas Científicas de América Latina, el Caribe, España y Portugal Proyecto académico sin fines de lucro, desarrollado bajo la iniciativa de acceso abierto 


\title{
DISCRIMINACIÓN SALARIAL POR GÉNERO EN MÉXICO
}

\author{
Jorge Eduardo Mendoza Cota* \\ Karina Jazmín García Bermúdez**
}

Fecha de recepción: 22 de octubre de 2008. Fecha de aceptación: 9 de febrero de 2009.

\section{Resumen}

Este estudio analiza la existencia de discriminación salarial por género en México en el año 2006. A partir de la teoría de capital humano se utiliza el método de OaxacaBlinder (1973) para realizar descomposiciones salariales. Con la aplicación de esta metodología se encuentra que debido a la discriminación salarial las mujeres perciben $12.4 \%$ menos de salario que los hombres con iguales características en términos de capital humano. En la región norte se estimó $7 \%$ de menores ingresos, para la región centro fue de $11.2 \%$ y la región sur presenta el nivel más alto que es de $18.3 \%$, corroborando de esta forma la situación de desigualdad de ingreso por género existente entre las regiones analizadas.

Palabras clave: mercado laboral, discriminación salarial, capital humano, retornos a la educación, distribución salarial.

* Investigador del Departamento de Estudios Económicos de El Colegio de la Frontera Norte. Correo electrónico: emendoza@colef.mx.

** Asistente de Investigador del departamento de Estudios Económicos de El Colegio de la Frontera Norte y profesora de la Universidad Iberoamericana de Tijuana. Correo electrónico: kgarcia@colef.mx. 


\section{Abstract}

The present work analyzes the existence of gender wage discrimination in Mexico in 2006. Starting from the human capital theory, the Oaxaca-Blinder (1973) method is used to carry out wage breakdowns. By applying this methodology we find that wage discrimination causes women to earn wages that are $12.4 \%$ less than do men with equal characteristics in human capital terms. In the north of Mexico wages are estimated to be $7 \%$ lower, while for the central region they were $11.2 \%$ less and in the south the difference was at its highest with women's wages $18.3 \%$ less than men's. This corroborates the situation of income inequality by gender that exists between the regions under analysis.

Key words: labor market, wage discrimination, human capital, returns to education, wage distribution.

\section{Résumé}

Cette étude analyse l'existence d'une discriminaton sexiste sur le plan des salaires au Mexique en 2006. Prenant pour base la théorie du capital humain, il est recouru à la méthode de Oaxaca-Blinder (1973) pour effectuer des décompositions salariales. En adoptant cette démarche on se rend compte que du fait de cette discrimination, les femmes perçoivent $12,4 \%$ de moins que les hommes avec les mêmes caractéristiques en termes de capital humain. Dans la région nord, on estime à $7 \%$ la différence, pour la région centre elle fut de 11,2\%, et la région sud présente la plus ample qui est de 18,3, ces chiffres confirmant la situation d'inégalité des revenus par genre entre les régions analysées.

Mots clés: marché du travail, discrimination sur le plan des salaires, capital humain, retours à l'éducation, ditribution des salaires.

\section{Resumo}

Este estudo analisa a existência da descriminação salarial por gênero no México no ano de 2006. A partir da teoria do capital humano se utiliza o método de Oaxaca-Blinder (1973) para realizar decomposições salariais. Com a aplicação dessa metodologia se encontra que devido à descriminação salarial as mulheres recebem $12.4 \%$ menos seu salário que os homens tendo características iguais nos termos de capital humano. Estimou-se na região norte $7 \%$ dos menores ingressos, na região centro foi de $11.2 \%$ e a região sul apresenta o nível mais alto que foi de $18.3 \%$, confirmando assim a situação de desigualdade do ingresso por gênero existente entre as regiões analisadas.

Palavra chave: mercado de trabalho, descriminação salarial, capital humano, retornos à educação, distribuição salarial.

\section{Desarrollo}




\section{Introducción}

mercado de trabajo es un espacio particular donde la mercancía es el trabajo
humano. Por ello, este mercado presenta diferentes aspectos sociales que deter-
minan impactos en el precio de la fuerza de trabajo, y que no son explicados en la lógica económica del mercado.

Entre los factores sociales, la discriminación es un fenómeno en el que un individuo o grupo es tratado de forma desfavorable, a causa de prejuicios, generalmente por pertenecer a una categoría social distinta. Entre los principales tipos de discriminación se encuentran el racismo y xenofobia, la homofobia, la discriminación a discapacitados y enfermos, la diferenciación según el estrato social, la discriminación religiosa y la discriminación a las mujeres.

Los estudios empíricos sobre la discriminación de género han encontrado evidencia empírica en varios países. Para el caso de España y Andalucía, García Pérez y Morales López (2006) encuentran que de $83 \%$ de las diferencias salariales medias, $17.3 \%$ puede ser consecuencia de la discriminación. En otro trabajo (Lago Peñas, 2002) se investiga la discriminación salarial por razones de género en el sector privado de España — integrando las teorías por el lado de la oferta y de la demanda-, además de descomponer la diferencia salarial entre sexos y cuantificar $18 \%$ del nivel de discriminación.

En otro trabajo Ying Chu (2007) estudia las diferencias salariales por género en la China urbana por región y sus cambios durante la primera década de la reforma económica (1988-1997). La descomposición del diferencial salarial por género que realiza revela que un bajo porcentaje del diferencial puede ser explicado por diferencias de género. Los resultados de sección cruzada dejan ver la posible existencia de discriminación por género, sobre todo a finales del periodo de reforma económica y desarrollo analizado.

En lo que respecta a América Latina, Abadía Alvarado (2005) analiza la existencia de discriminación estadística por sexo en el mercado laboral colombiano, sus resultados muestran que no hay evidencia de discriminación en el sector público, pero sí en el privado, sobre todo en el caso de aquellos individuos casados o en unión libre. En un trabajo realizado para Chile, Fuentes, Palma y Montero (2005) hacen un análisis de la discriminación salarial por género para el periodo 1990-2003. Encuentran que la discriminación salarial por género ha disminuido durante el periodo 1990-2003, pero aún sigue siendo positiva, situándose en 2003 en 27.5\%. En otro trabajo, Paredes y Rivero (1994) estudian la brecha salarial por género en Santiago entre 1985 y 1990. Los resultados muestran la presencia de discriminación en contra de la mujer en el 
mercado laboral, descubren que la discriminación por género en 1958 era de $91 \%$ ciento, mientras que para 1990 alcanzó 74\%. Montenegro (2001) realiza un análisis más detallado del fenómeno de la discriminación por género en Chile para los años 1990, 1992, 1994 y 1996, en el que aplica el método de regresiones de cuantiles y descompone la brecha salarial por género para toda la distribución de salarios. Encontró que el diferencial salarial no explicado es mayor en los cuantiles superiores de la distribución, lo que sugiere una mayor discriminación en contra de la mujer a medida que ésta tiene más años de escolaridad y de experiencia laboral. En un trabajo para Bolivia, Contreras y Galván (2002) examinan la discriminación salarial por género, etnia y su interacción en Bolivia para el periodo 1994-1999. Los resultados de la investigación muestran la existencia de discriminación salarial por género, etnia y su interacción.

Para el caso de los mercados de trabajo de México hay poca literatura relacionada con el tema de la discriminación salarial. En un trabajo de Rodríguez y Camberos (2007) se estiman las tasas de rendimiento de la educación en el mercado laboral de Hermosillo, Sonora, y realizan un análisis de la discriminación salarial por área de estudio profesional y género. Los autores encontraron diferencias salariales que no son explicadas por las diferencias en capital humano (educación), sino quizás sean más bien de carácter discriminatorio. Dichas diferencias son mayores en las ciencias económico-administrativas con $77.4 \%$, en ciencias sociales con $104 \%$, y finalmente en ingenierías y tecnológicas son menores con $50.5 \%$.

Aunque hay evidencia de que la mujer aún sufre de discriminación en el tipo de trabajo al que puede tener acceso, en la remuneración que obtiene, en las oportunidades de capacitación y de promoción, entre otros, es importante estimar el grado de discriminación en el ámbito nacional y por regiones. En este contexto, debe clarificarse el papel de la educación, la experiencia laboral u otros factores que se conjugan en la determinación de los salarios, tanto de hombres como de mujeres, para estimar el grado de discriminación salarial en el mercado laboral, con el fin de dirigir las políticas públicas en pos de mejorar dichos aspectos. Para cumplir con este propósito en este estudio se utiliza el método de descomposición de Oaxaca-Blinder (1973) para obtener la magnitud de la brecha salarial explicada por discriminación.

Los objetivos que se persiguen son los de obtener información actualizada sobre la discriminación salarial en México, analizando sobre todo si las diferencias salariales entre los hombres y mujeres que laboran se explican por factores de discriminación por género, y comparar la discriminación salarial en el ámbito nacional con los tres espacios geográficos específicos: la región formada por los estados de la frontera 
norte, la región de los estados del centro y la región de los estados del sur del país, ello en el año 2006.

En este trabajo las secciones se dividen en la presentación de la evidencia de la discriminación salarial en México, el enfoque teórico de los retornos a la educación y la discriminación salarial, la metodología de estimación de la discriminación salarial de género, los resultados de las estimaciones y finalmente las conclusiones del trabajo.

\section{Evidencia de discriminación salarial en México}

Con el fin conocer el ámbito de discriminación salarial en la economía mexicana, se analizó la Encuesta Nacional de Empleo Urbano (ENOE) de 2006 para determinar los niveles salariales, de educación y experiencia de la fuerza laboral que reciben ingresos monetarios por sueldos, salarios o jornal por su trabajo. ${ }^{1} \mathrm{El}$ total de la muestra seleccionada alcanzó un número de encuestados de 86,311 personas, $60 \%$ hombres y $40 \%$ mujeres, respectivamente. En el ámbito de región, las personas que recibieron ingresos por sueldos, salario y jornal son 15,246 (61\% hombres y $39 \%$ mujeres) en el norte; en el centro suman 29,399 (59\% hombres y 41\% mujeres), y 14,903 (61\% hombres y $39 \%$ mujeres) en el sur del país.

Ahora se presentan las características de las principales variables analizadas para determinar la discriminación salarial. En lo que respecta a la educación, entre las personas que reciben ingresos por salarios, las mujeres poseen mayor educación promedio en la muestra del ámbito nacional, con 11.1 años promedio de escolaridad formal comparados con los hombres que tienen 9.7. En las tres regiones estudiadas esta variable muestra un resultado muy similar (véase el cuadro 1 del anexo). En cuanto a la variable de experiencia laboral, los hombres que tienen una edad promedio mayor son al mismo tiempo los que poseen una mayor experiencia promedio. Algo que vale la pena resaltar es que comparado con la media nacional de 18.6 años de experiencia promedio, los hombres sobresalen en las regiones del norte y sur con 19.1 y 19 años respectivamente (véase el cuadro 2).

En relación con la variable ingresos, la retribución mensual promedio fue de 19,892 pesos para los hombres y 14,657 pesos para las mujeres en el ámbito nacional. Respecto a los ingresos por hora, el promedio fue de 126.5 pesos para los hombres y 121.3 pesos para las mujeres (véase el cuadro 3 ).

1 La ENOE cuenta con 319,332 entrevistas procesadas en el nivel individuo. 
$\mathrm{El}$ análisis por regiones ${ }^{2}$ muestra que la zona de la frontera norte cuenta con el ingreso mensual promedio más alto con 21,217 pesos para los hombres y 16,982 para las mujeres, mientras que la región sur es la que tiene el menor ingreso promedio mensual de 19,350 pesos para los hombres y 14,070 para las mujeres (véase el cuadro 3 ). En cuanto al ingreso por hora, se presenta la misma situación que con el salario mensual promedio.

Al considerar la variable profesión, los hombres que cuentan con nivel de educación profesionista recibieron 20,046 pesos mensuales en promedio y las mujeres recibieron 14,990, mientras que los hombres que no cuentan con este nivel de educación percibieron 19,735 pesos y las mujeres 14,484; es a partir de este nivel donde los ingresos aumentan a medida que crecen los años de educación, es decir, se observa más claramente una relación positiva entre los ingresos y la educación (véase el cuadro 4).

En las regiones centro y sur, las personas sin educación profesionista percibieron un mayor ingreso mensual promedio que las personas que cuentan con dicho nivel. Como ejemplo, en el centro los hombres profesionistas percibieron 19,506 pesos y las mujeres 14,198 , mientras que los hombres no profesionistas recibieron en promedio 19,831 pesos y las mujeres 14,264 (véase el cuadro 4).

La variable de género es un importante factor que influye en la determinación de los ingresos de los trabajadores en México. En general, los hombres percibieron mayores ingresos promedio tanto mensuales como por hora en todas las regiones analizadas, así como en el ámbito nacional. Por ello, se deduce que probablemente una de las razones de esta diferenciación se deba a que las habilidades adquiridas por medio de la educación formal no son valoradas en la misma magnitud entre hombres y mujeres. Así, a las mujeres el hecho de contar con más años de escolaridad no les implica una ventaja, pues los hombres perciben mayores ingresos. Además de estar directamente relacionadas las percepciones de los ingresos con los años de educación formal y género, hay otros factores que guardan relación con los ingresos, entre ellos la edad y el estado civil.

Por lo que toca a la edad, la muestra se dividió en tres grupos de edades. En el primero se consideró a las personas de 14 a 25 años, en el segundo a las de 26 a 40 años y en el tercero a las de 41 a 65 años de edad. Tomando en cuenta los salarios por hora promedio, se encontró que las mujeres muestran una tendencia más clara a recibir mayores salarios conforme aumenta su edad en las regiones estudiadas. Al analizar los salarios promedio mensuales, se observa que tanto hombres como mujeres perci-

2 La regionalización. 
ben una mayor remuneración salarial conforme aumenta la edad en todas las regiones analizadas. Sin embargo, la mujer recibe menor salario mensual promedio que los hombres en cada uno de los grupos de edad antes descritos (véase el cuadro 5).

El estado civil es otro de los factores que influyen en la determinación de los ingresos. De individuos que reciben ingresos por salarios, 44,151 son casados $(68 \%$ son hombres y $32 \%$ mujeres) y 33,247 son solteros ( $49 \%$ hombres y $51 \%$ mujeres). En el ámbito nacional, los hombres casados percibieron un salario mensual promedio de 20,899 pesos y las mujeres 15,763 ; los hombres solteros ganaron 18,037 pesos y las mujeres 13,731 (véase el cuadro 6).

El análisis por regiones muestra que la zona de la frontera norte cuenta con el ingreso mensual promedio más alto para las personas casadas: 22,531 pesos para los hombres y 17,970 para las mujeres; los hombres solteros perciben un ingreso promedio mensual de 18,800 pesos y las mujeres de 16,034, mientras que la región sur es la que tiene el menor ingreso promedio mensual, los hombres casados recibieron 19,851 pesos y las mujeres 15,682 , los hombres solteros percibieron un ingreso promedio mensual de 18,341 pesos y las mujeres de 12,776 (véase el cuadro 6).

Los resultados anteriores sustentan la hipótesis de que el género es uno de los factores que contribuye a agudizar el fenómeno de la discriminación salarial, pues sus efectos son distintos entre hombres y mujeres. Lo anterior ha tenido como resultado la intensificación del trabajo femenino dentro y fuera del hogar, la escasez de empleo y la disminución salarial.

A partir de los resultados del análisis realizado en este estudio se observa que en México el promedio de horas de trabajo doméstico y extradoméstico para las personas que reciben sueldo por salarios, según sexo, muestra en promedio lo siguiente: mujeres, 79 horas promedio trabajadas a la semana, mientras que en los hombres el promedio es de 65.75 horas. Lo que indica esta medición es que las mujeres trabajan en promedio 13.25 horas más a la semana que los hombres. Las mujeres continúan siendo las principales responsables de su familia, con un aumento del porcentaje de mujeres jefas del hogar, lo que les hace experimentar el conflicto trabajo-familia de forma especial.

La participación de las mujeres se asocia primordialmente con el trabajo asalariado, el trabajo por cuenta propia y el trabajo familiar sin pago. Analizar la estructura familiar de los hogares es fundamental para entender las desigualdades de género, ya que el tamaño, la composición y el tipo de hogares tienen diversas implicaciones en la vida de hombres y mujeres. 
Los indicadores seleccionados muestran que los diferenciales se van haciendo cada vez más acentuados a partir de los 15 años de edad, etapa que suele coincidir con el inicio de la fase reproductiva femenina. En contraste, al analizar la distribución de hombres y mujeres como adultos mayores en su posición familiar en los hogares, se comprueban las más amplias disparidades.

La mayoría de los promedios nacionales y regionales muestran que el nivel educativo de las mujeres ha igualado o hasta superado al de los hombres. Sin embargo, se observan importantes diferencias cuando la información se abre según distintas características de la población. Uno de los fenómenos que sintetiza la desigualdad en el mercado laboral es el hecho de que las mujeres ganan menos que los hombres. Las brechas entre las remuneraciones de mujeres y hombres son evidentes, incluso al controlar por horas trabajadas y nivel educativo. Las mujeres con mayor educación también son las que ganan significativamente menos que los hombres en esta misma condición. Por tanto, la evidencia encontrada sugiere la existencia de la discriminación en contra de la mujer en el mercado laboral.

\section{Enfoque teórico de la discriminación salarial}

\section{Teoría del capital humano}

El marco conceptual que permite vincular los conceptos de educación, experiencia e ingreso de los trabajadores en México se basa en el supuesto de la relación positiva entre los conocimientos y la productividad de los trabajadores. En este sentido, la educación es uno de los determinantes de los ingresos laborales. Además, la experiencia y la formación que adquieren las personas en el lugar de trabajo contribuyen al desarrollo de la calificación de la mano de obra, generando que se mejore la calidad del trabajo y el aumento de la productividad y, por tanto, de los ingresos de los trabajadores. En esta perspectiva, por el lado de la oferta, para que una persona decida invertir en educación adicional deberá estar compensada por mayores ingresos durante su ciclo de vida, lo cual se evalúa por el valor presente de los rendimientos a la educación (Becker, 1993). ${ }^{3}$

3 Los rendimientos a la educación se formalizan de la siguiente manera:

$$
V=\frac{1}{r}\left[\left(Y_{s}-I_{c}\right)\left(1-e^{-r t}\right)+Y_{s+c}\left(1-e^{-r t}\right)\right]
$$

donde: $Y_{s}=$ son los rendimientos asociados a la educación, $I_{c}=$ costo de oportunidad del tiempo dedicado a adquirir habilidades en el trabajo, fuera de la educación formal, $Y_{s+c}=$ rendimientos asociados a la educación y a la experiencia y $\mathrm{V}$ es el valor presente de los rendimientos futuros de la educación. 
Por ello, con objeto de percibir mayores ingresos los trabajadores más educados deben tener una mayor productividad, es decir, que la productividad marginal de los trabajadores debe aumentar con el nivel educativo, la experiencia y la capacitación. Por tanto, las inversiones en capital humano no son sólo los desembolsos en educación formal y no formal como la capacitación y adiestramiento, sino también los constituyen los gastos en salud, la crianza de los hijos durante la edad preescolar, la búsqueda de empleo, la migración y los gastos en información para localizar mejores opciones de empleo.

Según Spence (1973), aquellos individuos que cuenten con mayores niveles educativos tendrán mayores incentivos para invertir en educación, ya que ésta es un mecanismo de información con que cuentan los empleadores para tomar la decisión de escoger a un posible trabajador para determinado puesto de trabajo.

\section{Teoría de discriminación en el mercado de trabajo}

La teoría de discriminación en el mercado de trabajo de Becker (1971) considera a la discriminación como un prejuicio personal que implica no asociarse con miembros de un grupo particular. En el caso del género, la discriminación afecta a la contratación de mujeres para trabajos que no se ajustan a la distribución de papeles entre sexos en una sociedad. Dado que en condiciones iguales los empresarios prefieren contratar a hombres (la contratación de mujeres llevaría aparejado un costo no económico derivado de su inaceptabilidad social), en situaciones de amplia discriminación las mujeres deben aceptar salarios más bajos que los varones para tener acceso a trabajos idénticos.

Becker muestra cómo el dinero o el ingreso es una medida clara de la discriminación. Si un individuo tiene preferencia por discriminar tendremos que suponer que actuará como si estuviese dispuesto a pagar algo, ya sea de manera directa o mediante la reducción de su ingreso, para asociarse económicamente con ciertas personas en lugar de hacerlo con otras. Según este autor, se puede sostener que hombres y mujeres de la misma edad y de la misma escolaridad pueden obtener el mismo ingreso siempre y cuando no exista la discriminación.

En esta perspectiva, se distingue entre el cálculo racional económico, el prejuicio y los estigmas sociales, siendo los dos últimos fenómenos sociales de la discriminación. Por ello, la discriminación no se puede eliminar mediante la disponibilidad de la información y de la educación. En este contexto, los salarios de las personas pueden diferir entre trabajadores con iguales habilidades en el mismo trabajo simplemente 
por su raza, género, nacionalidad de origen, orientación sexual y otras características que no corresponden a capacidades de las personas relacionadas de manera directa con el trabajo que realizan. Por lo general, estas diferencias son atribuidas a discriminación en el mercado de trabajo.

\section{Formalización de la discriminación salarial por género}

La discriminación existe en el mercado de trabajo por razones de género cuando dos individuos con la misma calificación son tratados de un modo distinto sólo por su género (Blau, Ferber y Winkler, 1998). Si además está extendida y es persistente, el propio comportamiento de las mujeres se verá adversamente afectado: su incentivo para invertir en capital humano será menor que en el caso de los hombres. La interrelación de los conceptos antes referidos se puede desarrollar a partir de la siguiente función propuesta por Mincer (1974), aplicada a muestras de corte transversal:

$$
\operatorname{Lny}_{i}=\alpha+\beta_{1} S_{1}+\beta_{2} X_{i}+\beta_{3} X_{i}^{2}+u_{i}
$$

Donde a, $\beta_{1}, \beta_{2}>0$ y $\beta_{3}<0$ y $L n y_{i}=$ logaritmo natural del ingreso, $\mathrm{S}=$ educación, $\mathrm{X}=$ experiencia, $\mathrm{X}^{2}=$ experiencia al cuadrado, $\beta_{1}=$ tasa de retorno a la educación, $\beta_{2}$ y $\beta_{3}=$ miden la tasa de retorno a la experiencia.

Como argumenta Mincer (1974), la importancia que tienen los coeficientes de esta ecuación radica en que cuantifican el efecto que tiene el stock de capital humano sobre el nivel y la distribución de los ingresos laborales. A partir de esa función se puden estimar ecuaciones de salarios para hombres (h) y otra para mujeres (m). Por tanto:

Donde $\mathrm{Y}=$ vector columna de los ingresos, $\mathrm{X}=$ matriz de variables independientes, $\beta=$ vector columna de coeficientes a estimar $\mathrm{y} u=$ términos de perturbación.

$$
\begin{aligned}
& Y_{h}=X_{h}^{\prime}+u_{h} \\
& Y_{m}=X_{m}^{\prime}+u_{m}
\end{aligned}
$$

Una vez estimados los retornos a la educación, es posible aplicar el método de Oaxaca-Blinder para realizar descomposiciones salariales por género y determinar de esa forma qué parte del diferencial se debe a discriminación y qué parte se debe a diferencias en dotación:

$$
Y_{h}-Y_{m}=\left(X_{h}^{\prime}-X_{m}^{\prime}\right) \hat{\beta}_{h}+\left(\hat{\beta}_{h}-\hat{\beta}_{m}\right) X_{m}^{\prime}
$$

Donde: 
$\bar{Y}_{h}-\bar{Y}_{m}=$ diferencia en los salarios medios de hombres y mujeres.

$\left(\bar{X}_{h}^{\prime}-X_{m}^{\prime}\right) \hat{\beta}_{h}=$ corresponde a las diferencias en dotación.

$\left(\hat{\beta}_{h}-\hat{\beta}_{m}\right) X_{m}^{\prime}=$ es la discriminación existente.

El término anterior refleja una diferencia en la compensación de las variables de capital humano en el mercado laboral según el género. De acuerdo con Oaxaca (1973), en un mercado laboral libre de discriminación, este último elemento debiese ser idéntico para hombres y mujeres. Es decir, las diferencias salariales existentes obedecerían únicamente a diferencias en la dotación de capital humano de los trabajadores. En caso que exista discriminación, el premio o retorno de un año adicional de escolaridad es mayor o menor para distintos tipos de personas, siendo la diferencia el factor de discriminación.

\section{Aspectos metodológicos}

\section{El modelo empírico de capital humano}

Para examinar la validez empírica de la diferencia salarial entre hombres y mujeres, inicialmente se especificó una ecuación econométrica para estimar los retornos a la educación tanto para los hombres como para las mujeres:

$$
\text { Lny }_{i}=\alpha+\beta_{1} E d u c+\beta_{2} \operatorname{Exp}+\beta_{3} \operatorname{Exp}_{i}^{2}+\beta_{4} \operatorname{Pr} \text { of }+u_{i}
$$

Donde $\alpha, \beta_{1}, \beta_{2}>0$ y $\beta_{3}<0$

Lny $=$ logaritmo natural del ingreso por hora

Educ $=$ educación

Exp $=$ experiencia

$\operatorname{Exp}^{2}=$ experiencia al cuadrado

Prof $=$ profesionista

$\mathrm{u}=$ términos de error

Esta ecuación nos dice que el logaritmo del ingreso individual es una función de la educación, la experiencia y la experiencia al cuadrado que captura la concavidad de la función de ingresos así como la variable $(\mathrm{P})$ profesionista. La importancia que tienen los coeficientes de esta ecuación radica en que cuantifican el efecto que tiene el stock de capital humano sobre los ingresos y sobre la distribución de los ingresos laborales (Mincer, 1974). 


\section{Modelo de estimación de la discriminación salarial}

El cálculo de la brecha de ingresos entre géneros se efectúa aplicando la técnica de A. Blinder (1973) y R. Oaxaca (1973) a la función antes mencionada, la cual se fundamenta en dos supuestos: en primer lugar, se asume que todos los individuos tienen iguales habilidades y, segundo, se asume que enfrentan similares oportunidades. Ello implica que un año de educación es rentable para todos los trabajadores y en todas las ocupaciones en la misma magnitud.

Como se mencionó antes, se deben modelar los salarios de los hombres y mujeres por separado en función de su capital humano, para luego descomponer la diferencia salarial promedio en dos elementos: uno que representa las diferencias en la dotación del capital humano de ambos grupos (es decir, las variables que explican la productividad del trabajo), y otro que indica una diferencia en el pago que hace el mercado por la posesión de dicha dotación (diferencias en el retorno o premio al capital humano). En un mercado laboral libre de discriminación, este último elemento debiese ser idéntico para hombres y mujeres. Es decir, las diferencias salariales existentes obedecerían sólo a diferencias en la dotación de capital humano de los trabajadores (Oaxaca, 1973).

Para estimar la descomposición, se midieron las variables por sus valores medios en la muestra y se calculó la diferencia en $L n y^{H}-L n y^{M}$, es decir, los salarios de hombres y mujeres. Una parte de la diferencia en el logaritmo de los salarios puede deberse al nivel de educación en promedio, la experiencia o el tipo de industria $u$ ocupación en la que se encuentran los trabajadores. Al sustituir en el lado derecho de la ecuación de salarios estimada obtenemos:

$$
\begin{aligned}
L n y^{H}-L n y^{M} & =\hat{\beta}_{0}^{H}+\hat{\beta}_{1}^{H} E d u c^{H}+\hat{\beta}_{2}^{H} \operatorname{Exp}^{H}+\hat{\beta}_{3}^{H} \operatorname{Pr} o f^{H} \\
& -\left(\hat{\beta}_{0}^{M}+\hat{\beta}_{1}^{M} E d u c^{M}+\hat{\beta}_{2}^{M} \operatorname{Exp}^{M}+\hat{\beta}_{3}^{M} \operatorname{Pr} o f^{M}\right) \\
& =\left(\hat{\beta}_{0}^{H}-\hat{\beta}_{0}^{M}\right)+\left(\hat{\beta}_{1}^{H}-\hat{\beta}_{1}^{M}\right) E d u c^{H}+\left(\hat{\beta}_{2}^{H}-\hat{\beta}_{2}^{M}\right) \operatorname{Exp}^{H}+\left(\hat{\beta}_{3}^{H}-\hat{\beta}_{3}^{M}\right) \operatorname{Pr} o f^{H} \\
& +\hat{\beta}_{1}^{H}\left(E d u c^{H}-E d u c^{M}\right)+\hat{\beta}_{2}^{M}\left(\operatorname{Exp}^{H}-\operatorname{Exp}^{M}\right)+\hat{\beta}_{3}^{M}\left(\operatorname{Pr} o f^{H}-\operatorname{Pr} o f^{M}\right)
\end{aligned}
$$

Los tres últimos términos del lado derecho capturan las diferencias en salarios explicadas por diferencias en educación, experiencia y ocupación, diferencias no atribuibles a "discriminación salarial", más bien representan diferencias en "productividad". Los cuatro términos restantes: 


$$
\left(\hat{\beta}_{0}^{H}-\hat{\beta}_{0}^{M}\right)+\left(\hat{\beta}_{1}^{H}-\hat{\beta}_{1}^{M}\right) E d u c^{H}+\left(\hat{\beta}_{2}^{H}-\hat{\beta}_{2}^{M}\right) \operatorname{Exp}^{H}+\left(\hat{\beta}_{3}^{H}-\hat{\beta}_{3}^{M}\right) \operatorname{Pr} o f^{H}
$$

Representan las diferencias salariales explicadas por diferencias en los retornos a la educación $\hat{\beta}_{1}^{H}-\hat{\beta}_{1}^{M}$, diferencias en pago por experiencia $\hat{\beta}_{21}^{H}-\hat{\beta}_{2}^{M}$, diferencias en pago por profesión $\hat{\beta}_{3}^{H}-\hat{\beta}_{3}^{M}$ y diferencias en "salarios iniciales" (la diferencia en interceptos representa diferencias en salarios si no hubo educación, ni experiencia y los trabajadores no fueron profesionales). Esta diferencia salarial no explicada es interpretada usualmente como diferencias discriminatorias.

El segundo bloque representa el cálculo de la discriminación existente, pues refleja una diferencia en la compensación de las variables de capital humano en el mercado laboral según el género, revelada por las diferencias en los coeficientes estimados. En caso que exista discriminación, los hombres por cada año adicional de escolaridad adquirido serían premiados en el mercado laboral en una magnitud mayor que las mujeres, es decir, para un mismo activo (igual cantidad de años de escolaridad) el pago a los hombres sería mayor.

La muestra se seleccionó con base en el concepto de asalariados (obreros, empleados, jornaleros), además se incluyen los ingresos laborales por horas trabajadas. La educación se define por los años de escolaridad formal, considerando los años de escolaridad de manera sucesiva hasta llegar a los estudios de posgrado. El género está definido como una variable dicotómica en donde el hombre toma el valor de uno, y cero la mujer. La profesión está definida como una variable dictómica en donde las personas con un nivel profesionista toman el valor de uno y cero las personas que no cuentan con este nivel. La experiencia se calcula por la edad menos los años de educación menos seis años. La edad de los individuos comprendida es de los 14 a los 65 años. Dado que en el estado civil existen varias categorías, para fines de estimación se conjuntaron dentro de una sola variable a aquellos que se encuentran casados o en unión libre, asignándoles el número uno; y se unió también a las personas solteras, separadas, divorciadas o viudas, asignándoles el valor de cero.

Respecto a la regionalización, de las 32 entidades federativas en primer lugar se toma a la población total del país y, en segundo lugar, se ha dividido el territorio nacional en tres regiones: en la norte se consideran los seis estados de la frontera norte: Baja California, Sonora, Chihuahua, Coahuila, Nuevo León y Tamaulipas; en la región centro: Aguascalientes, Guanajuato, San Luis Potosí, Querétaro, Estado de México, Distrito Federal, Morelos, Hidalgo, Puebla y Tlaxcala; y en la región sur: Guerrero, Oaxaca, Chiapas, Veracruz y Tabasco, para hacer una comparación relativa en la percepción de los ingresos por trabajo dado el género de las personas. 
Esta regionalización permite conocer de manera general si las diferentes regiones de México son homogéneas en cuanto a los ingresos de los trabajadores mujeres y hombres en el mercado laboral, o si existen diferencias derivadas de los cambios económicos regionales experimentados en México como resultado de la apertura económica y de la firma del Tratado de Libre Comercio de América del Norte (TLCAN). Tanto los estados del norte de México como los del centro han venido modificando su dinámica económica, lo que ha determinado una mayor expansión del sector manufacturero en el norte, mientras que el centro se ha especializado en los sectores de servicios y comercio. Se espera que la regionalización permita saber si estos cambios han repercutido también en el mercado laboral de hombres y mujeres en México.

\section{Análisis de las estimaciones de discriminación salarial por género}

Con el fin de estimar los coeficientes de las tasas de retorno a la educación para hombres y mujeres, que se requieren para obtener la descomposición salarial, se llevaron a cabo estimaciones con un modelo de mínimos cuadrados generalizados aplicados a una base de datos de corte transversal. ${ }^{4}$ Las pruebas estadísticas del modelo aplicado al ámbito nacional y a las diferentes regiones sugieren que la ecuación empírica parece estar adecuadamente especificada, en la medida que la $\mathrm{R}$ cuadrada ajustada fue superior a $80 \%$ en todos los casos.

Los resultados muestran retornos a la educación superiores para los hombres en la región del norte. Además, el coeficiente de los profesionistas hombres muestra que éstos tendieron a obtener mayores ingresos que las mujeres en el centro y el sur. Finalmente, en el ámbito nacional las mujeres exhibieron mayores retornos a la ecuación que los hombres, aunque en términos de la experiencia los hombres tuvieron mayores retornos. Lo anterior sugiere la presencia de factores no previstos en el modelo que podrían estar sesgando las estimaciones, debido a la imposibilidad de las mujeres de participar y de permanecer en el mercado laboral debido a aspectos relacionados con el matrimonio y el cuidado de los hijos.

Con el fin de explicar los resultados estadísticos de las dos regresiones, se efectuó una regresión Probit de participación laboral con el fin de obtener información de la muestra total de mujeres tanto económicamente activas como no activas. Los resultados muestran que el coeficiente de educación tiene signo positivo; a medida que aumentan los años de educación formal la probabilidad crece el retorno (véase

4 Se utilizó el método de mínimos cuadrados generalizados para evitar problemas de autocorrelación y heterocedasticidad que arrojaron el estadístico Durbin Watson y la prueba de White, respectivamente. 
el cuadro 8). El coeficiente correspondiente a la edad resultó positivo, lo que indica que a medida que aumenta la edad de la mujer crece su probabilidad de participar en el mercado laboral, aunque el incremento con cada año adicional de edad en la mujer resulta decreciente tal como lo demuestra el signo negativo de esta variable al cuadrado. No obstante, el coeficiente correspondiente al estado conyugal resultó negativo, lo que indica que las mujeres casadas tienen una menor probabilidad de participar en el mercado laboral. Además, es posible observar que la variable sobre los hijos tiene signo negativo; a medida que aumenta el número de hijos, la probabilidad disminuye. De igual manera sucede con las variables referentes a las horas dedicadas al cuidado de niños y quehaceres del hogar sin pago. Finalmente, las horas dedicadas al trabajo también son un factor que limita la participación de la mujer en el mercado laboral.

De los resultados anteriores es posible considerar que existe una proporción importante de mujeres que encuentren limitaciones para tener acceso al mercado laboral, como contar con experiencia y capacitación laboral, lo cual podría estar generando un sesgo de selección que refleja la imposibilidad de distinguir el nivel de retornos por el cual estarían dispuestas a trabajar las mujeres que no participan en el mercado de trabajo. Por esta razón es probable que - aunque existe evidencia estadística de discriminación salarial derivada de los ingresos promedio de las mujeres y los hombres- la ecuación de retornos no la capture más que parcialmente. A continuación se estima la descomposición de diferencias de salarios incorporando los promedios de ingreso de hombres y mujeres para corroborar si existe dicha discriminación en los ingresos.

\section{Análisis de la discriminación salarial por género}

En el presente trabajo fue posible comprobar que para el caso de México existe una brecha entre los ingresos percibidos por hombres y mujeres que trabajan. Los resultados de los cálculos muestran la existencia de discriminación salarial por género. De esta manera, de acuerdo con las estimaciones de la descomposición salarial en el ámbito nacional, la diferencia entre ingresos de hombres y mujeres sólo es explicada en $87.6 \%$ por la dotación de capital humano y $12.4 \%$ por la estructura del mercado.

Por su parte, la descomposición de esta diferencia en la región norte, aclarada por las diferencias en la dotación capital humano entre hombres y mujeres, es de 93\%, mientras que $7 \%$ se explica por la estructura del mercado. En la región centro la descomposición de esta diferencia muestra que $88.8 \%$ está descrita por la dotación de capital humano y $11.2 \%$ está relacionada con la estructura de mercado. Finalmente, 
en la región sur la descomposición de esta diferencia muestra que $81.7 \%$ está explicado por la dotación de capital humano y $18.3 \%$ se vincula a la estructura del mercado (véase el cuadro 9).

De esta manera, es posible señalar que en el presente estudio los resultados de los cálculos sobre las diferencias salariales muestran evidencia de la existencia de discriminación salarial por género tanto en el ámbito nacional como en las regiones en que fue dividida la nación. En particular, se destaca la evidencia de una mayor discriminación salarial en la región sur del país, seguida del centro y finalmente en la región norte se aprecia un menor nivel de diferencias salariales no explicadas por las diferencias en dotación de capital humano, que en buena medida pueden ser atribuidas a la discriminación. Cabe destacar que los aspectos relacionados con la estructura del mercado laboral parecen ser determinantes en la estructura de la discriminación salarial y generar un sesgo de las estimaciones de los coeficientes de retornos a la educación.

\section{Conclusiones}

En el presente trabajo, al utilizar la descomposición salarial de Blinder y Oaxaca, se presenta evidencia de discriminación salarial por género en el mercado laboral de la economía de México y en las regiones que la conforman en el año de 2006, que deriva de la existencia de un premio salarial por género para los hombres. Así pues, en el análisis realizado a las variables que influyen en la determinación de salarios se encontró que tanto en el ámbito nacional como regional los hombres son los que representan el mayor porcentaje en la muestra (alrededor de 60\%). Además, en general los hombres recibieron mayores ingresos promedio tanto mensuales como por hora en cada una de las regiones estudiadas, así como en el nivel nacional. En lo referente a educación, las mujeres son las que poseen el promedio de años de instrucción más alto que el de los hombres, con 11.1 y 9.7 años en promedio en cada caso, respecto al ámbito nacional. En cada una de las regiones analizadas se encontró una situación muy similar. En cuanto a la experiencia, los hombres son los que presentan una mayor experiencia promedio de 18.6 años en el ámbito nacional, 19.1 años en la región norte y 19 en la región sur del país.

$\mathrm{Al}$ analizar los ingresos de hombres y mujeres en el ámbito nacional se encontró que el salario promedio mensual de las mujeres representa $74 \%$ del que reciben los hombres. En cada una de las regiones se observa la misma relación entre los salarios de mujeres y hombres, pero en la región norte es donde las personas percibieron un salario mayor y la región sur es donde percibieron los menores salarios, recibiendo 
las mujeres en el norte $80 \%$ del salario de los hombres y en el sur $72 \%$. Al tomar en cuenta la variable profesión, en el país las personas que cuentan con este nivel de preparación reciben el mayor salario promedio tanto mensual como por hora.

Sin embargo, las mujeres siguen en desventaja, las profesionistas obtienen $75 \%$ del salario promedio mensual que perciben los hombres en dicha categoría y en lo que respecta a las personas que no cuentan con estudios profesionales, las mujeres reciben $73 \%$ del salario mensual promedio que ganan los hombres. En el análisis por regiones se encuentra una situación muy similar. El estado civil es otro de los factores que influyen en la determinación de los ingresos. De los individuos analizados, se observó que las personas casadas perciben un salario mayor que el que reciben las solteros, hay más hombres casados que mujeres, pero éstas reciben $75 \%$ del salario promedio mensual que el de los hombres. Y de la categoría de solteros, las mujeres perciben $76 \%$ del salario promedio mensual del que obtiene los hombres.

Por último, se observa que al considerar el promedio de horas de trabajo doméstico y extradoméstico para las personas que reciben sueldo por salarios, las mujeres trabajan en promedio 13.25 horas más a la semana que los hombres. Por lo que se corrobora que las mujeres continúan siendo las principales responsables de su familia, con un aumento del porcentaje de mujeres jefas del hogar, lo que les hace experimentar el conflicto trabajo-familia de forma especial.

Mediante la aplicación de la metodología desarrollada por Oaxaca y Blinder, y aun cuando existe probabilidad de que exista sesgo de selección, se encuentra que la discriminación salarial en el ámbito nacional es de $12.4 \%$, es decir, las mujeres perciben $12.4 \%$ menos de salario que los hombres con iguales características en términos de capital humano. Del mismo modo, en la región norte se sitúa en 7\%, para la del centro es de $11.2 \%$ y la del sur presenta el nivel más alto que es de $18.3 \%$, corroborando de esta forma la situación de desigualdad de ingreso existente entre las regiones analizadas. Los resultados sugieren que las regiones norte y centro - con una mayor dinámica económica basada en las manufacturas- y el sector servicios presentan una menor discriminación salarial, mientras que el sur mantiene una posición relativa desventajosa.

El problema de la discriminación salarial y sus efectos en el mercado laboral se relacionan con el retorno esperado de la inversión en capital humano, pues cuando éste es menor, las mujeres, al decidir cuánta escolaridad deben adquirir, escogerán un nivel inferior al óptimo. Ello en el largo plazo mermará su capacidad de generación de ingresos y se constituye como una barrera para una asignación eficiente de recursos dentro de una economía. Por otro lado, se deben mencionar los efectos que tendrá 
esta situación sobre la acumulación de ingresos para la edad de jubilación, pues el aporte que se realiza en las cuentas de capitalización individual depende del salario percibido y, al ser éste menor, el ahorro también será menor.

Por ello, la discriminación en el mercado laboral mexicano es un tema que debe ser tomado más en cuenta en la discusión de la política pública para determinar los factores que están influyendo en la generación de esta situación. En el presente estudio se presentó evidencia de la existencia de este problema, pero se debe continuar con la tarea de determinar el origen de éste, debido a que la existencia de discriminación salarial puede tener consecuencias, incluso antes del momento de la entrada al mercado laboral, que es cuando la persona efectivamente percibirá menores ingresos.

\section{Bibliografía}

Acosta, Elaine, Marcela Perticara y Claudio Ramos, "Oferta laboral femenina y cuidado infantil", Banco Interamericano de Desarrollo, BID, agosto de 2005.

Ashfelter, Orley Ashenfelter y Ronald Oaxaca, "The Economics of Discrimination: Economists Enter the Courtroom", The American Economic Review, vol. 77, núm. 2, Papers and Proceedings of the Ninety-Ninth Annual Meeting of the American Economic Association, 1987, pp. 321-325.

Becker, Gary, Human Capital. A Theoretical and Empirical Analysis with Special Reference to Education, Chicago, The University of Chicago Press, 1993.

, The Economics of Discrimination, Chicago, segunda edición, University of Chicago Press, 1971.

, "Investment in Human Capital: A Theoretical Analisis", Journal of Political Economy, 70, 1962, pp. 9-49.

Bernal, Rosa, Clotilde Charlot y Anne-Marie Urban, "Enfoque de género en programas y proyectos de desarrollo", Banco Interamericano de Desarrollo, BID, 2002.

Blaug, Mark, "Educación y empleo", Instituto de Estudios Económicos, Madrid, 1981.

, "The Empirical Status of Human Capital Theory: A Slightly Jaundiced Survey", Journal of Economic Literature, 14, 1976, pp.827-855.

Blau, Francine, Marianne Ferber y Anne Winkler, The Economics of Women, Men and Work, Upper sadle River, Prentice-
Hall, 3a edición, 1998.

Blinder, Alan, "Wage Discrimination: Reduced Form and Structural Variables", Journal of Human Resources, 8, 1973, pp. 436-455.

Collins, Randall, La sociedad credencialista/ Sociología histórica de la educación y de la estratificación, Madrid, Akal, 1989.

Contreras, Dante y Marco Galván, "Are The Gender And Ethnic Wage Discrimination Decreasing In Bolivia? Evidence of 19941999", UDAPE, 2002.

Chu, Ng Ying, "Gender earnings differentials and regional economic development in urban China, 1988-1997", Review of Income and Wealth, Hong Kong Baptist University, Serie 53, núm. 1, 2007.

Dickens, William y Kevin Lang, "A Test of Dual Labor Market Theory", American Economic Review, vol. 75, núm. 4, 1985, pp. 792-805.

Einsentein, Zillah, "Developing a Theory of Capitalist Patriarchy and Socialist Feminism", Monthly Review Press, New York, 1979, pp. 5-55.

Fuentes, Jeannette, Amalia Palma, Rodrigo Montero, "Discriminación salarial por género en Chile: una mirada global." Estudios de Economía , 2005, 32 (2), pp. 133-57.

García, José I. y María Morales, "Discriminación salarial en el mercado de trabajo español con especial referencia al caso de Andalucía", Centro de Estudios Andaluces, Documento de Trabajo Serie Economía E2006/18, 2006. 
Heckman, James, "Sample Selection Bias as a Specification Error", Econométrica, 47, 1979, pp. 153-162.

Interpretación de la de la Declaración Americana de los Derechos y Deberes del Hombre, dentro del marco de los artículos 64 de la convención Americana sobre Derechos Humanos, 14 de julio de 1989, Serie A, núm. 10, párrafos 35-45.

Karime, Luz y Abadía Alvarado, “¿Por qué ha disminuido el desempleo?", Revista Javeriana, vol. 719, 2005, pp.26 - 33.

Lago, Ignacio, "La discriminación por razones de genero: un análisis empírico del sector privado en España", Revista Española de Investigaciones Sociológicas, núm. 98, 2002, pp. 171-196.

Mendoza, Jorge E., J, "Educación, experiencia y especialización manufacturera en la frontera norte de México", Comercio Exterior, vol. 52, núm. 4, 2002.

Mincer, Jacob, Schooling, Experiencie and Earnings, NuevaYork, Columbia University Press, 1974.

Montenegro, Claudio, "Wage Distribution in Chile: Does Gender Matter? A Quantile
Regression Approach", The World Bank, Poverty Reduction and Economic Management Network, 2001.

Paredes, Ricardo y Luis Rivero, Gender Wage Diferentials in Chile, 1958-1990, Santiago de Chile, mimeo, 1994.

Oaxaca, Ronald, "Male-Female Wage Differentials in Urban Labor Markets", International Economic Review, núm. 14, 1973, pp. 693-709.

y Ransom, M., "Identification in Detailed Wage Decompositions", The Review of Economics and Statistics, vol. 81, núm. 1, 1999, pp. 154-157.

Rodríguez, Reyna y Mario Camberos, "Análisis de la discriminación salarial de la mujer en Hermosillo, Sonora", Política y Cultura, UAM-Xochimilco, México, 2007, pp. 225256.

Schultz, Theodore, "Investment in Human Capital", American Economic Review, núm. 51, pp. 1-17, 1961.

Spence, Spence, "Job Market Signaling", Quarterly Journal of Economics, 87, 1973 pp. 355-374. 
Anexo

Cuadro 1

Escolaridad promedio según región y género

\begin{tabular}{lrrr}
\hline Región & Estadísticas descriptivas & \multicolumn{1}{c}{ Hombres } & \multicolumn{1}{c}{ Mujeres } \\
\hline \multirow{3}{*}{ Nacional } & Media & 9.7 & 11.1 \\
& D.S. & 4.7 & 4.8 \\
\multirow{4}{*}{ Norte } & Obs. & 51,623 & 34,688 \\
& Media & 9.9 & 11.1 \\
& D.S. & 4.4 & 4.5 \\
Centro & Obs. & 9,312 & 5,934 \\
& Media & 9.6 & 10.8 \\
& D.S. & 4.5 & 4.8 \\
Sur & Obs. & 17,403 & 11,996 \\
& Media & 9.9 & 11.3 \\
& D.S. & 5.0 & 4.9 \\
& Obs. & 9,017 & 5,886 \\
\hline
\end{tabular}

Fuente: Elaboración propia con base en el Instituto Nacional de Estadística, Geografía e Informática, Encuesta Nacional de Ocupación y Empleo, 2006.

Cuadro 2

Experiencia laboral según región y género

\begin{tabular}{lrrr}
\hline Región & Estadísticas descriptivas & \multicolumn{1}{c}{ Hombres } & \multicolumn{2}{c}{ Mujeres } \\
\hline \multirow{3}{*}{ Nacional } & Media & 18.6 & 17.4 \\
& D.S. & 13.2 & 12.5 \\
\multirow{3}{*}{ Norte } & Obs. & 51,623 & 34,688 \\
& Media & 19.1 & 17.8 \\
& D.S. & 13.2 & 12.6 \\
Centro & Obs. & 9,312 & 5,934 \\
& Media & 18.5 & 17.5 \\
& D.S. & 13.1 & 12.6 \\
\multirow{2}{*}{ Sur } & Obs. & $17,403.0$ & $11,996.0$ \\
& Media & 19.0 & 17.0 \\
& D.S. & 13.4 & 12.3 \\
& Obs. & $9,017.0$ & $5,886.0$ \\
\hline
\end{tabular}

Fuente: Elaboración propia con base en el Instituto Nacional de Estadística, Geografía e Informática, Encuesta Nacional de Ocupación y Empleo, 2006. 
Cuadro 3

Ingresos laborales mensuales y por hora según región y género

\begin{tabular}{|c|c|c|c|c|c|}
\hline \multirow[b]{2}{*}{ Región } & \multirow[b]{2}{*}{ descriptivas } & \multicolumn{2}{|c|}{ Mensuales } & \multicolumn{2}{|c|}{ Por hora } \\
\hline & & Hombres & Mujeres & Hombres & Mujeres \\
\hline Nacional & $\begin{array}{c}\text { Media } \\
\text { D.S. } \\
\text { Obs. } \\
\text { Media } \\
\text { D.S. }\end{array}$ & $\begin{array}{r}19,892.0 \\
28,831.3 \\
51,623 \\
21,216.7 \\
36,077.5\end{array}$ & $\begin{array}{r}14,657.3 \\
21,061.2 \\
34,688 \\
16,982.4 \\
19,444.1\end{array}$ & $\begin{array}{r}126.5 \\
255.5 \\
51,623 \\
128.6 \\
230.4\end{array}$ & $\begin{array}{r}121.3 \\
226.0 \\
34,688 \\
134.9 \\
268.1\end{array}$ \\
\hline Cen tro & $\begin{array}{c}\text { Obs. } \\
\text { Media } \\
\text { D.S. } \\
\text { Obs. } \\
\text { Media } \\
\text { D.S. } \\
\text { Obs. }\end{array}$ & $\begin{array}{r}9,312 \\
19,875.1 \\
25,485.9 \\
17,403 \\
19,350.2 \\
26,259.4 \\
9,017\end{array}$ & $\begin{array}{r}5,934 \\
14,378.9 \\
18,497.9 \\
11,996 \\
14,069.8 \\
22,562.3 \\
5,886\end{array}$ & $\begin{array}{r}9,312 \\
125.0 \\
209.7 \\
17,403 \\
119.7 \\
204.4 \\
9,017\end{array}$ & $\begin{array}{r}5,934 \\
121.2 \\
208.8 \\
11,996 \\
109.1 \\
197.5 \\
5,886\end{array}$ \\
\hline
\end{tabular}

Fuente: Elaboración propia con base en el Instituto Nacional de Estadística, Geografía e Informática, Encuesta Nacional de Ocupación y Empleo, 2006.

Cuadro 4

Ingresos laborales por mes, por región según género y profesión

\begin{tabular}{rcrrrr}
\hline \multirow{2}{*}{ Región } & & \multicolumn{2}{c}{ Profesionista } & \multicolumn{2}{c}{ No Profesionista } \\
\hline \multirow{3}{*}{ Nacional } & Estadísticas descriptivas & \multicolumn{1}{c}{ Hombres } & \multicolumn{1}{c}{ Mujeres } & \multicolumn{1}{l}{ Hombres } & \multicolumn{1}{c}{ Mujeres } \\
& Media & $20,046.40$ & $14,990.72$ & $19,734.51$ & $14,483.66$ \\
& D.S. & $21,220.56$ & $16,487.76$ & $29,556.61$ & $21,698.85$ \\
\multirow{3}{*}{ Norte } & Obs. & 3,925 & 3351 & 41219 & 27,169 \\
& Media & $24,814.79$ & $18,867.73$ & $20,733.96$ & $16,655.15$ \\
& D.S. & $27,765.41$ & $16,549.73$ & $36,729.57$ & 19768.95 \\
\multirow{3}{*}{ Centro } & Obs. & 636 & 544 & 7,784 & 4,922 \\
& Media & $19,506.13$ & $14,198.13$ & $19,831.45$ & $14,263.69$ \\
& D.S. & $18,537.27$ & $10,699.62$ & $26,050.97$ & $19,136.27$ \\
\multirow{2}{*}{ Sur } & Obs. & 1,021 & 882 & 13425 & 8,992 \\
& Media & $17,728.07$ & $13,861.84$ & $19,520.02$ & $14,008.49$ \\
& D.S. & $19,094.88$ & $12,958.98$ & $27,296.29$ & $24,065.67$ \\
& Obs. & 927 & 770 & 6,892 & 4,414 \\
\hline
\end{tabular}

Fuente: Elaboración propia con base en el Instituto Nacional de Estadística, Geografía e Informática, Encuesta Nacional de Ocupación y Empleo, 2006. 
Cuadro 5

Ingresos laborales promedio por hora según región por grupos de edad y género

\begin{tabular}{|c|c|c|c|c|c|c|c|}
\hline \multirow{2}{*}{ Región } & \multirow{2}{*}{$\begin{array}{l}\text { Estadísticas } \\
\text { descriptivas }\end{array}$} & \multicolumn{4}{|c|}{$\begin{array}{l}\text { Grupos de edad } \\
26 \text { a } 40 \text { años }\end{array}$} & \multicolumn{2}{|c|}{41 a 65 años } \\
\hline & & Hombres & Mujeres & Hombres & Mujeres & Hombres & Mujeres \\
\hline \multirow{3}{*}{ Nacional } & Media & $18,195.88$ & $12,368.25$ & $20,297.12$ & $14,766.62$ & $21,086.73$ & $16,555.05$ \\
\hline & D.S. & $26,854.76$ & $18,730.17$ & $29,464.65$ & $20,610.88$ & $29,841.26$ & $23,359.79$ \\
\hline & Obs. & 14,121 & 8,409 & 18,095 & 13,501 & 13,912 & 9,365 \\
\hline \multirow{3}{*}{ Norte } & Media & $17,822.95$ & $14,600.96$ & $22,345.83$ & $17,582.74$ & $22,905.24$ & $18,148.91$ \\
\hline & D.S. & $30,207.40$ & $15,746.63$ & $33,716.64$ & $20,261.88$ & $42,945.60$ & $20,883.80$ \\
\hline & Obs. & 2,480 & 1,457 & 3,370 & 2,348 & 2,731 & 1,766 \\
\hline \multirow{3}{*}{ Centro } & Media & $18,314.89$ & $12,363.43$ & $20,253.38$ & $14,266.12$ & $21,049.43$ & $16,442.31$ \\
\hline & D.S. & $23,422.50$ & $16,231.45$ & $27,386.20$ & $16,325.82$ & $24,838.40$ & $22,727.00$ \\
\hline & Obs. & 4,619 & 2,834 & 5,852 & 4,283 & 4,251 & 3,002 \\
\hline \multirow{3}{*}{ Sur } & Media & $18,622.69$ & $11,434.33$ & $19,251.51$ & $14,132.62$ & $20,149.68$ & $16,377.72$ \\
\hline & D.S. & $28,578.57$ & $28,098.07$ & $25,983.72$ & $19,803.12$ & $24,274.74$ & $20,601.80$ \\
\hline & Obs. & 2,319 & 1,394 & 3,163 & 2,382 & 2,501 & 1,527 \\
\hline
\end{tabular}

Fuente: Elaboración propia con base en el Instituto Nacional de Estadística, Geografía e Informática, Encuesta Nacional de Ocupación y Empleo, 2006.

Cuadro 6

Ingresos laborales por mes por región según género y estado civil

\begin{tabular}{lcrrrr}
\hline \multirow{2}{*}{ Región } & \multicolumn{2}{c}{ Casado } & \multicolumn{2}{c}{ Soltero } \\
\hline \multirow{3}{*}{ Nacional } & Estadísticas descriptivas & \multicolumn{1}{c}{ Hombres } & \multicolumn{1}{c}{ Mujeres } & \multicolumn{1}{c}{ Hombres } & \multicolumn{1}{c}{ Mujeres } \\
& Media & $20,899.08$ & $15,763.35$ & $18,037.83$ & $13,731.47$ \\
& D.S. & $30,802.02$ & $22,550.36$ & $24,689.25$ & $19,682.52$ \\
\multirow{4}{*}{ Norte } & Obs. & 29,898 & 14,253 & 16,228 & 17,019 \\
& Media & $22,531.06$ & $17,970.48$ & $18,800.18$ & $16,034.25$ \\
& D.S. & $39,539.97$ & $21,686.08$ & $28,487.74$ & $16,967.17$ \\
Centro & Obs. & 5,558 & 2,728 & 3,023 & 2,843 \\
& Media & $20,923.19$ & $15,411.14$ & $17,840.46$ & $13,621.25$ \\
& D.S. & $26,578.89$ & $20,867.43$ & $23,084.90$ & $16,505.19$ \\
Sur & Obs. & 9,719 & 4,283 & 5,002 & 5,836 \\
& M edia & $19,851.73$ & $15,682.28$ & $18,340.59$ & $12,776.18$ \\
& D.S. & $26,727.97$ & $24,773.61$ & $25,265.73$ & $20,538.26$ \\
\hline
\end{tabular}

Fuente: Elaboración propia con base en el Instituto Nacional de Estadística, Geografía e Informática, Encuesta Nacional de Ocupación y Empleo, 2006. 
Cuadro 7

Estimación de retornos a la educación para México,

en los niveles nacional y regiones, norte, centro y sur

\begin{tabular}{|c|c|c|c|c|c|c|c|c|}
\hline \multirow[b]{2}{*}{ Variable } & \multicolumn{2}{|c|}{ Nacional } & \multicolumn{2}{|c|}{ Norte } & \multicolumn{2}{|c|}{ Centro } & \multicolumn{2}{|c|}{ Sur } \\
\hline & Hombres & Muieres & Hombres & Mujeres & Hombres & Mujeres & Hombres & Mujeres \\
\hline \multirow{2}{*}{ Constante } & $4.046^{*}$ & $3.674^{*}$ & $4.102^{\star}$ & $4.021^{*}$ & $4.166^{*}$ & $3.857^{\star}$ & $3.829^{\star}$ & $3.135^{*}$ \\
\hline & -217.265 & -163.449 & -102.575 & -83.167 & -130.242 & -104.306 & -78.505 & -49.872 \\
\hline \multirow[t]{2}{*}{$\begin{array}{l}\text { Años de } \\
\text { educación }\end{array}$} & $0.016^{*}$ & $0.029^{\star}$ & $0.026^{\star}$ & $0.023^{*}$ & $0.0073^{\star}$ & $0.016^{\star}$ & $0.014^{\star}$ & $0.05^{*}$ \\
\hline & -10.602 & -17.812 & -8.059 & -6.369 & -2.8 & -6.039 & -3.724 & -11.029 \\
\hline \multirow[t]{2}{*}{ Profesionista } & $0.101^{*}$ & $0.104^{*}$ & 0.038 & $0.115^{\star}$ & $0.141^{*}$ & $0.123^{\star}$ & $0.176^{*}$ & $0.163^{\star}$ \\
\hline & -6.136 & -6.47 & -0.954 & -3.057 & -4.562 & -4.204 & -4.864 & -4.461 \\
\hline \multirow[t]{2}{*}{ Experiencia } & $0.007^{\star}$ & $0.014^{*}$ & $0.0063^{*}$ & $0.0085^{\star}$ & $0.0053^{\star}$ & $0.011^{\star}$ & $0.0117^{*}$ & $0.0200^{*}$ \\
\hline & -16.124 & -25.848 & -6.72 & -7.84 & -6.877 & -12.386 & -10.844 & -14.694 \\
\hline$R^{2}$ ajustado & 0.853 & 0.842 & 0.872 & 0.857 & 0.848 & 0.852 & 0.852 & 0.836 \\
\hline Durbin - Watson & 1.71 & 1.8 & 1.72 & 1.74 & 1.7 & 1.8 & 1.8 & 1.84 \\
\hline
\end{tabular}

Fuente: Elaboración propia. Valores t estadísticos entre paréntesis. *: significativo al 1\%; **: significativo al 5\%.

Cuadro 8

Resultados de las estimaciones del modelo Probit de participaci de la mujer en el mercado laboral en el nivel nacional

\begin{tabular}{lccc}
\hline \multicolumn{1}{c}{ Variable } & Coeficientes & Estadistico $t$ & Prob. \\
\hline C & $-0.9663^{\star}$ & -6.3542 & 0.0000 \\
Edad & $0.1189^{\star}$ & 12.2988 & 0.0000 \\
Edad2 & $-0.0015^{\star}$ & -11.2823 & 0.0000 \\
Educación & $0.0594^{*}$ & 15.5061 & 0.0000 \\
Yiefe & 0.0332 & 0.0000 & 1.0000 \\
Estado conyugal & $-0.3185^{\star}$ & -7.5160 & 0.0000 \\
Hijos & $-0.0440^{\star}$ & -3.7953 & 0.0001 \\
Horas cuidado de niños & -0.0003 & -1.0001 & 0.3173 \\
Horas dedicadas al hogar & $-0.0039^{\star}$ & -9.6893 & 0.0000 \\
\hline
\end{tabular}

Fuente: Elaboración propia con base en el Instituto Nacional de Estadística, Geografía e Informática, Encuesta Nacional de Ocupación y Empleo, 2006.

Cuadro 9

Resultados de la descomposición de las diferencias salariales

\begin{tabular}{lrrrrrr}
\hline \multicolumn{1}{c}{ Region } & \multicolumn{1}{c}{ Dif. total Lny } & \multicolumn{1}{c}{ \% } & \multicolumn{1}{c}{ Dif. explicada } & \multicolumn{1}{c}{ Dif. discriminación } & \multicolumn{2}{c}{$\%$} \\
\hline Nacional & 0.095 & 100 & 0.083 & 87.6 & 0.012 & 12.4 \\
Norte & 0.049 & 100 & 0.046 & 93 & 0.0033 & 7 \\
Centro & 0.102 & 100 & 0.09 & 88.8 & 0.012 & 11.2 \\
Sur & 0.114 & 100 & 0.092 & 81.7 & 0.022 & 18.3 \\
\hline
\end{tabular}

Fuente: Elaboración propia con base en el Instituto Nacional de Estadística, Geografía e Informática, Encuesta Nacional de Ocupación y Empleo, 2006. 